\title{
Review Article \\ Extracellular Vesicles as Biomarkers of Systemic Lupus Erythematosus
}

\author{
Javier Perez-Hernandez ${ }^{1,2}$ and Raquel Cortes ${ }^{1,2}$ \\ ${ }^{1}$ Genotyping and Genetic Diagnosis Unit, INCLIVA Biomedical Research Institute, Accesorio 4, Avenida Menendez Pelayo, \\ 46010 Valencia, Spain \\ ${ }^{2}$ Research Group of Cardiometabolic and Renal Risk, INCLIVA Biomedical Research Institute, Accesorio 4, Avenida Menendez Pelayo, \\ 46010 Valencia, Spain
}

Correspondence should be addressed to Raquel Cortes; raquel.cortes@uv.es

Received 9 August 2015; Accepted 25 August 2015

Academic Editor: Giuseppe Murdaca

Copyright (C) 2015 J. Perez-Hernandez and R. Cortes. This is an open access article distributed under the Creative Commons Attribution License, which permits unrestricted use, distribution, and reproduction in any medium, provided the original work is properly cited.

\begin{abstract}
Systemic lupus erythematosus is an autoimmune disease that predominantly affects women and typically manifests in multiple organs. The damage caused by this disorder is characterized by a chronic inflammatory state. Extracellular vesicles (EVs), including microvesicles (also known as microparticles), apoptotic bodies, and exosomes, are recognized vehicles of intercellular communication, carrying autoantigens, cytokines, and surface receptors. Therefore, the evidence of EVs and their cargo as biomarkers of autoimmune disease is rapidly expanding. This review will focus on biogenesis of extracellular vesicles, their pathophysiological roles, and their potential as biomarkers and therapeutics in inflammatory disease, especially in systemic lupus erythematosus.
\end{abstract}

\section{Introduction}

Systemic lupus erythematosus (SLE) is an autoimmune disease, characterized by its clinical heterogeneity and effect on several organs since it has a wide profile of autoantibodies $[1,2]$. The prevalence of SLE varies from 20 to 150 cases per 100,000 of population, with a high prevalence in women $(9: 1)$ [3]. Despite being currently incurable, in recent decades, survival rates and longevity have increased due to improvements in therapies and diagnosis. Thus, it has a high impact on longterm medical costs associated with frequent cycles of disease flare and remission [4].

As are many autoimmune disorders, SLE is a multifactorial disease in which genetic and environmental factors interact to modulate the final phenotype. Some loci have been associated with an increase in the risk of SLE (complement components $\mathrm{Clq}$ and $\mathrm{C} 4$ ) while others are generally related to several autoimmune diseases, such as diabetes or rheumatoid arthritis (e.g., PTPN22 and STAT4) [5, 6]. Moreover, an epigenetic dysregulation, found in many SLE patients, has been proposed as crucial in the initiation and progression of the disease. Thus, several studies concerning DNA methylation $[7,8]$, histone acetylation $[9,10]$, and microRNAs [11, 12] have evidenced epigenetic cross talk [13]. Furthermore, environmental factors (Epstein-bar virus and pesticides) and hormones may trigger autoimmune responses and modulate the alternating periods of SLE flares [14].

One of the most affected organs in SLE is the kidney. The deposition of immune complexes, activation of complements and macrophages, and production of proinflammatory cytokines and chemokines lead to lupus nephritis (LN). Present in almost two-thirds of SLE patients during their lifetime $[15,16]$, up to $30 \%$ of patients progress to endstage renal failure [17]. In particular, clinical manifestations of active LN include proteinuria, active urinary sediments, and progressive renal dysfunction [18]. Currently, the invasive procedure of renal biopsy provides a direct visualization of renal affection. A recent work, however, shows no correlation between clinical and histological remission, which discards this procedure as a prognostic biomarker [19]. 
TABLE 1: Key features of extracellular vesicles.

\begin{tabular}{lclll}
\hline EV types & Size & Biogenesis & Markers & Contents \\
\hline Exosomes & 30-100 nm & $\begin{array}{l}\text { Endolysosomal pathway. } \\
\text { Released by exocytosis of } \\
\text { multivesicular bodies }\end{array}$ & $\begin{array}{l}\text { Tetraspanins (CD63, } \\
\text { CD9, and CD81), Alix, } \\
\text { and TSG101 }\end{array}$ & $\begin{array}{l}\text { miRNAs and mRNA; lipids, } \\
\text { DNA membrane proteins } \\
\text { and lipids, cytokine } \\
\text { receptors, and MHC } \\
\text { molecules }\end{array}$ \\
\hline Microvesicles/microparticles & $100-1000 \mathrm{~nm}$ & $\begin{array}{l}\text { Cell surface. Outward } \\
\text { budding of plasma } \\
\text { membrane }\end{array}$ & $\begin{array}{l}\text { Integrins, selectins, and } \\
\text { CD40 ligand }\end{array}$ & $\begin{array}{l}\text { mRNA, noncoding RNAs, } \\
\text { membrane proteins, } \\
\text { receptors, and cytoplasmic } \\
\text { proteins }\end{array}$ \\
\hline Apoptotic bodies & Up to $5000 \mathrm{~nm}$ & $\begin{array}{l}\text { Cell surface. Released from } \\
\text { cellular blebs during } \\
\text { apoptosis }\end{array}$ & Phosphatidyl-serine & $\begin{array}{l}\text { Nuclear fractions, cell } \\
\text { organelles, DNA, rRNA, } \\
\text { and mRNA }\end{array}$ \\
\hline
\end{tabular}

EV: extracellular vesicles, MHC: major histocompatibility complex, mRNA: messenger RNA, miRNA: microRNA, rRNA: ribosomal RNA, and TSG101: tumor susceptibility gene 101.

Despite being well established and easy to measure, complements $\mathrm{C} 3$ and $\mathrm{C} 4$, proteinuria, anti-dsDNA, or creatinine clearance is not as specific or as sensitive as desired. Currently, the SLE Disease Activity Index (SLEDAI) is the most commonly used indicator. It consists of a list of 24 items of which sixteen are clinical variables and eight are laboratory tests such as urinalysis, blood complement levels, increased anti-DNA antibody levels, and low platelet and white blood cell counts. A final score of 6 or higher seems to be consistent with an active disease state [20].

Despite improvements in the diagnosis and prevention of SLE flares, laboratory markers are still unsatisfactory. Over the last few years, the extracellular vesicles (EV), which carry nucleic acids, proteins, and lipids, have been described as essential players in several cellular processes [21, 22]. EVs are small membranous vesicles, ranging from $30 \mathrm{~nm}$ to $5 \mu \mathrm{m}$, and receive different names depending on their biogenesis and origin. Usually, they are classified as exosomes, microvesicles/microparticles, and apoptotic bodies.

This review focuses on the role of extracellular vesicles $(\mathrm{EV})$ as biomarkers to assess disease activity and the response to therapy in SLE.

\section{Extracellular Vesicle Biogenesis and Characteristics}

Extracellular vesicles, small membranous spherical structures composed of a lipid bilayer, are released by different kind of cells and found such biofluids as urine, plasma, saliva, CSF, synovial fluid, and breast milk [23]. These vesicles can be released by different kinds of cells and carry DNA, coding and noncoding RNAs, proteins, and lipids [21, 22]. Profiling of EV-associated RNA has shown important differences with parental cellular RNA [24]. Moreover, RNA species (miRNA or mRNA) shuttled by EVs maintain their function when transferred to the recipient cells, suggesting epigenetic signaling and an important role in cell-to-cell communication [25].

The general term "EV" includes different types of vesicles. They are not homogeneous and overlapping in size and are classified according to different parameters, biochemical composition, morphology, biogenesis, and size $[26,27]$
(Table 1). Exosomes are the smallest vesicles $(30 \mathrm{~nm}$ to $150 \mathrm{~nm}$ in diameter), derive from the inward budding of endosomes, and accumulate in intraluminal vesicles known as multivesicular bodies. These EVs are released to the lumen by exocytosis [28]. Microvesicles or microparticles (also referred to as shedding vesicles, ectosomes, or prostasomes) are generally larger than exosomes $(100 \mathrm{~nm}$ to $1000 \mathrm{~nm}$ ) and include all structures created by budding and fission directly from the plasma membrane [29]. Finally, apoptotic bodies are released as the consequence of apoptosis, and their diameters vary from $1000 \mathrm{~nm}$ to $5000 \mathrm{~nm}$. They are also produced by direct budding of the membrane when cells suffer apoptosis (Table 1).

Currently, there is no consensus on a gold-standard method to isolate/purify EVs according to the type of biofluid or EV type desired [30]. That, notwithstanding, differential ultracentrifugation is the method most frequently mentioned in the literature. It is based on a two-step protocol that begins with a low-speed centrifugation at 10,000-17,000 $\times$ g, which separates apoptotic bodies and larger EVs, and a second step at a higher speed, $100,000-200,000 \times \mathrm{g}$, depending on the study and the average size of EV required [31]. Alternatively, EVs can be isolated using immunoaffinity beads against surface proteins, filtering the sample through a nanomembrane, utilizing commercial products for exosome enrichment, or employing size-exclusion chromatography principles [3235].

According to the International Society for Extracellular Vesicles (ISEV), most EV preparations are heterogeneous. Over the last few years, there have been great efforts made to establish appropriate guidance for EV isolation and characterization with minimum experimental requirements [30]. Furthermore, three public databases contain updated information about EVs: EVpedia, ExoCarta, and Vesiclepedia [36-38].

\section{Extracellular Vesicles in Inflammatory Disease}

The damage caused by most autoimmune disorders is characterized by a chronic inflammatory state; so the regulation 


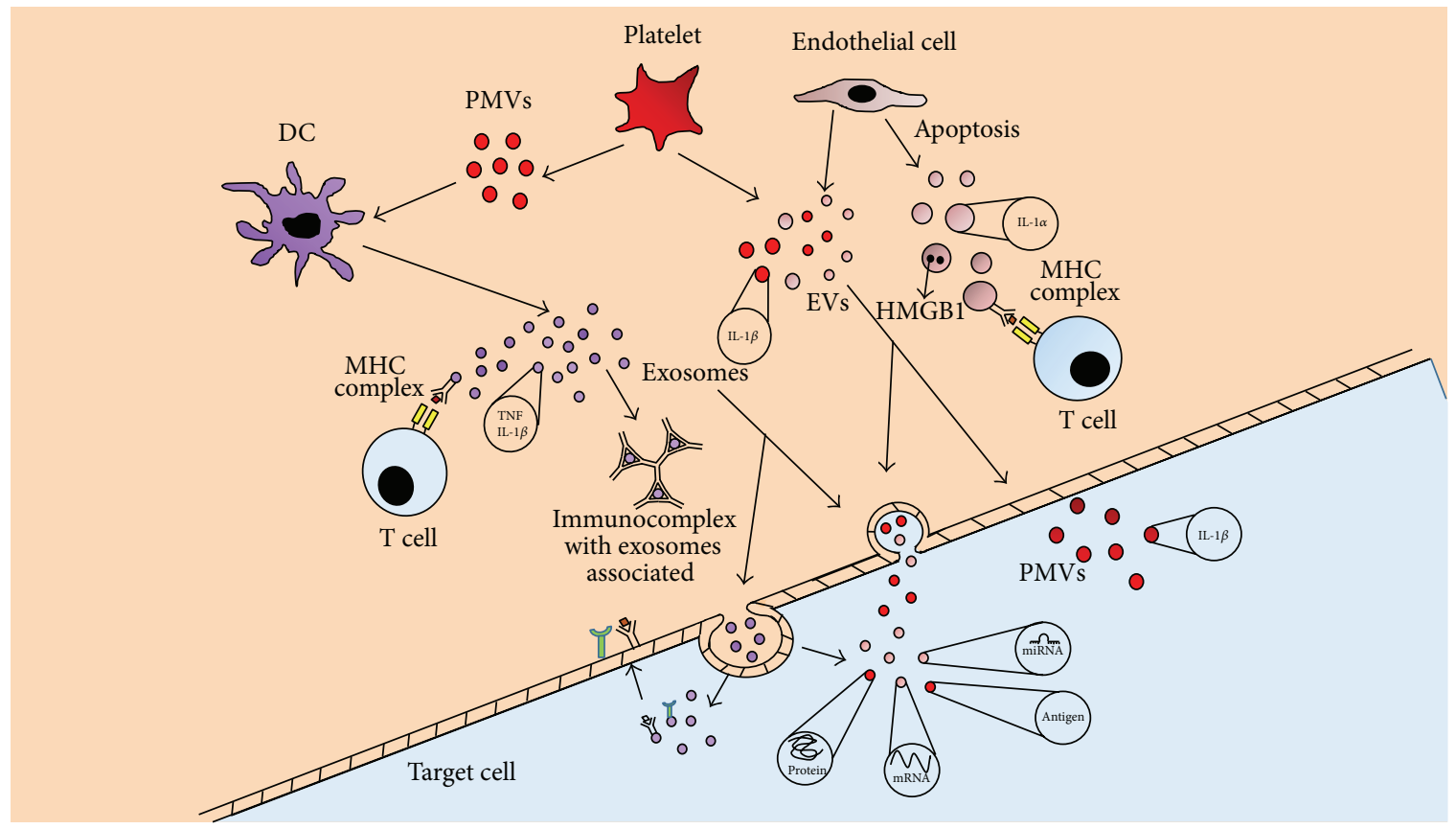

FIGURE 1: Role of extracellular vesicles in inflammation. Extracellular vesicles (EVs) from mature dendritic cells (DC) provide antigen to T cells and promote a proinflammatory response, mediated by host factors present within exosomes and apoptotic bodies (TNF, HMGB1, etc.). Autoantigens in EVs are recognized by autoantibodies and form immune complexes. Platelet-derived microvesicles (PMVs) activate DC and carry IL-1 $\beta$. EVs in target cell can be involved in antigen presentation and the transfer of major histocompatibility complex (MHC) molecules and antigens, participating in immune regulation. Finally, EVs activate or transfer surface receptors and deliver various RNA species (including mRNA and small RNAs) to target cells. DC: dendritic cell, EVs: extracellular vesicles, MHC: major histocompatibility complex, and PMVs: platelet-derived microvesicles.

of inflammation becomes essential in order to ameliorate a patient's condition.

Recently, few studies have been conducted in order to establish the relationship between damage-associated molecular patterns (DAMPs) and EV transport. These are endogenous molecules found normally inside cells such as histones, purine metabolites, and mitochondrial components. Under cellular stress or injury conditions, however, DAMPs are released into the extracellular space by damaged tissues, thus activating innate immune cells [39]. Therefore, they are likely to play a determinant role in the appearance and persistence of inflammation. Some DAMPs have been characterized inside EVs as nuclear HMGB1, high mobility group protein B1, ATP (inside apoptotic bodies), or S100 proteins (group of ligands of toll-like receptors) (Figure 1) [40, 41].

Moreover, the EV transport of cytokines and chemokines has emerged as an interesting mechanism for the spread and maintenance of inflammation. For instance, rheumatoid arthritis patients present platelet-derived microparticles with an abundance of IL- $1 \beta$, which induces cytokine release from synovial fibroblasts [42]. Other authors have shown apoptotic bodies carrying active forms of CX3CL1/fractalkine and stimulating chemotaxis in macrophages [23, 43].

Similarly, microvesicles found in the synovial fluid of rheumatoid arthritis patients form proinflammatory immune complexes which may work as autoantigens and autoadjuvants, initiating and perpetuating autoantibody production
(Figure 1) [44, 45]. Moreover, in juvenile idiopathic arthritis, synovial exosomes released by macrophages transport a nuclear phosphoprotein named DEK. This protein, involved in chromatin organization, tends to form high affinity complexes with IgG2, which results in joint inflammation [46].

Many studies have pointed out the defective clearance of apoptotic bodies and their subsequent accumulation as a main source of autoantigens in SLE. This results in both chronic organ and tissue damage, as well as the development and maintenance of the systemic autoimmune disease [47, 48]. Recently, circulating microparticles of SLE patients have been associated with particular clinical features, and specific protein patterns have been found. This suggests the importance of EV in driving pathological responses [49-51]. In addition, major ribonucleoproteins antigenically active for lupus and Sjogren's syndrome have been found in salivary gland-derived exosomes [52].

\section{Extracellular Vesicles as Biomarkers of Systemic Lupus Erythematosus}

The study of circulating microparticles (MP) in the plasma of SLE patients has outlined novel subpopulations of platelet, endothelial, and leukocyte-derived MP, some of which have clinical and serological correlations. Cytometry studies performed by Nielsen et al. showed correlations between a population of MP of endothelial origin (AnxV-CDMPs) and 
disease activity measures, glomerulonephritis, and vascular dysfunction [49]. Thus, the phenotype of endothelial MP offers strong potential as a specific biomarker of vascular pathology associated with SLE. Confirming this hypothesis, Parker et al. have shown an increase of endothelial MP with active SLE when compared to controls. Immunosuppressive therapy reduced the cardiovascular risk by reducing the number of circulating endothelial MP [53].

Moreover, the protein signature of these MP reveals specific patterns that could be used as biomarkers of the activity and progression of SLE. Østergaard et al. have shown a special spectrum of MP in SLE patients, with a particularly unbalanced and decreased microtubule and cytoskeletal composition, which differs from healthy individuals or even rheumatoid arthritis patients [50]. Therefore, the amounts and characteristics of circulating MPs provide new targets for assessing SLE pathogenicity and treatments.

Nevertheless, lupus nephritis (LN) is still a major cause of the morbidity and mortality of SLE with $10-30 \%$ of all cases progressing to end-stage renal disease [17]. The investigation of new biomarkers to assess glomerular damage without invasive biopsy has become essential in order to monitor disease progression.

In that sense, urine is the ideal biological fluid for new biomarkers because of its uncomplicated and noninvasive collection. Since the identification and characterization of urinary exosomes by Pisitkun et al., 2004 [54], many studies focusing on urinary exosomes as a source of biomarkers in renal, systemic, and urogenital diseases have been performed [55-58].

Some EV-associated miRNAs, small noncoding RNAs that modulate gene expression, have been proposed as biomarkers of kidney damage in SLE. Over the last few years, the characterization of exosomal miRNA, as opposed to nonexosomal miRNA, by deep sequencing has confirmed the notion of urinary exosomes as a stable source of miRNA biomarkers [59]. Particularly, Ichii et al. have shown an increase in the levels of miR-26a in exosomes from patients of LN and a positive correlation with urinary protein levels, suggesting its convenience as a predictive biomarker of podocyte injury [60]. Similarly, Solé et al. have reported reduced levels of miR-29c in LN patients when compared to controls. Moreover, those levels correlated with renal function and the degree of renal fibrosis, highlighting a potential role in predicting histological fibrosis [61].

Especially relevant is the study of miR-146a, reported to be markedly downregulated in PBMCs, contributing to alterations in type 1 interferon (IFN) pathway in patients with SLE [62]. Furthermore, several studies have assessed its biomarker relevance and found it downregulated as well in plasma and serum $[63,64]$ but overexpressed in the glomeruli of LN patients [65]. Moreover, some SNPs have been correlated with a lower expression of miRNA in a casecontrol study in Europeans [66]. Finally, our group has found much higher levels of miR-146a inside urinary exosomes compared to whole urine or exosome-depleted fractions, especially in patients with active LN (data under review).

Regarding messenger RNA cargo, similar studies have been performed to find new markers of kidney damage.
Recently, urinary exosome levels of CD2AP mRNA (protein participating in the glomerular filtration barrier) were found downregulated in patients and correlated with proteinuria and severity of renal fibrosis [67]. Similarly, the induction of podocyte damage in rats showed an increase of cystatin C mRNA levels in exosomes, which was representative of glomerular damage, correlating with renal mRNA and protein expression [68].

Finally, protein levels of the adhesion molecule ADAM10 were found higher in the exosomes of patients with glomerular disease, including LN [69]. An important substrate of this protein is the Notch receptor, not only involved in podocyte development but also playing a role in glomerular disease [70]. Moreover, transcription factors related to early podocyte injury were found in urinary EVs, but not in the whole urine of acute and chronic renal patients [57].

Thus, the analysis of urinary exosomes could be considered a reliable, noninvasive approach to the physiological state, offering complementary information to the invasive kidney biopsies. Exosomes are likely to replace biopsies in the future.

\section{Extracellular Vesicles as a Therapeutic Approach}

Leaving aside their promising future as biomarkers, EVs and their cargo could be exploited for therapeutic purposes in a broad range of procedures.

Due to the fundamental role of EVs in regulating biological processes and promoting inflammation and tumor growth under pathophysiological conditions [71], therapeutic actions are being developed to reduce the load of circulating EVs using different strategies: by inhibiting EV formation and release, by blocking EV-specific components with small interfering RNA, and by inhibiting EV uptake $[72,73]$. Although reducing the amount of apoptotic bodies or MPs is especially attractive for autoimmune disorders with a high inflammatory component such as SLE, interfering with the general aspects of biogenesis could lead to undesirable offtarget effects. Therefore, such actions would require a targeting system capable of selecting EV-cell specific populations.

Nevertheless, there has been a rapid increase in the number of studies investigating the role of EVs in the modulation of the immune system. Thus, EVs containing antiinflammatory substances could be used as therapeutic agents to promote immunosuppressive responses. Some studies have shown these types of compounds to have a longer half-life when encapsulated in EVs, increasing the survival of mice after LPS-induced septic shock [74]. Therefore, these vesicles could work as immunomodulatory agents for autoimmune, inflammatory, and hypersensitivity disorders $[23,75]$.

Several studies have been performed in order to examine the immunomodulatory action of dendritic cell (DC) derived exosomes. For instance, bone marrow-derived DCs were treated with IL-10, and the reduction of autoimmunity was evaluated in some murine models of disease, such as collagen-induced arthritis and delayed hypersensitivity [76, 77]. Further work, however, will be required before clinical 
translation is possible in order to optimize cell types for the production of well-defined EV therapeutic agents that are safe in the long run.

Regarding drug delivery, patient-derived EVs could be used to package molecules capable of avoiding immune responses. Exosomes are ideal for transfer purposes and may become a strong delivery tool for pharmacological agents. Because of their bilipidic structure, they are flexible vectors with the ability to carry select nucleic acids (miRNA, siRNA, and mRNA), proteins, and active chemical drugs across biological barriers $[74,78,79]$.

Altogether, this information underscores the broad potential of EV for the treatment and prevention of flares in autoimmune disorders like SLE. Nonetheless, further investigation is needed to elucidate the precise effect of $\mathrm{EV}$ treatment with immunomodulatory purposes.

\section{Concluding Remarks}

Extracellular vesicles have emerged as important "nanoshuttles" of information between cells, carrying proteins, genetic information, and bioactive lipids to modify the phenotype and function of recipient cells. EVs are potential regulators in autoimmune disorders, having a determinant role in the appearance and maintenance of inflammation. In SLE, the defective clearance of apoptotic bodies and their accumulation represents a major source of autoantigens. The presence of EV-specific patterns and their cargo as biomarkers of SLE activity and progression is rapidly expanding. In that sense, miRNAs, mRNA, and proteins transported into urinary exosomes are representative of glomerular damage, correlating with proteinuria and the severity of renal fibrosis in lupus nephritis. Still, the precise pathophysiological functions of these vesicles and their role as therapeutic agents or targets are not fully understood. Although further studies are necessary, we foresee a great potential for EVs as immunomodulatory agents and therapeutic vehicles in the future.

\section{Conflict of Interests}

The authors declare that there is no conflict of interests regarding the publication of this review.

\section{References}

[1] H. A. Al-Shobaili, A. A. Al Robaee, A. A. Alzolibani, and Z. Rasheed, "Antibodies against 4-hydroxy-2-nonenal modified epitopes recognized chromatin and its oxidized forms: role of chromatin, oxidized forms of chromatin and 4-hydroxy2-nonenal modified epitopes in the etiopathogenesis of SLE," Disease Markers, vol. 33, no. 1, pp. 19-34, 2012.

[2] T. Colasanti, A. Maselli, F. Conti et al., "Autoantibodies to estrogen receptor $\alpha$ interfere with T lymphocyte homeostasis and are associated with disease activity in systemic lupus erythematosus," Arthritis \& Rheumatism, vol. 64, no. 3, pp. 778787, 2012.

[3] G. C. Tsokos, "Systemic lupus erythematosus," The New England Journal of Medicine, vol. 365, no. 22, pp. 2110-2121, 2011.
[4] T. Li, G. S. Carls, P. Panopalis, S. Wang, T. B. Gibson, and R. Z. Goetzel, "Long-term medical costs and resource utilization in systemic lupus erythematosus and lupus nephritis: a fiveyear analysis of a large medicaid population," Arthritis Care \& Research, vol. 61, no. 6, pp. 755-763, 2009.

[5] K. L. Moser, J. A. Kelly, C. J. Lessard, and J. B. Harley, "Recent insights into the genetic basis of systemic lupus erythematosus," Genes and Immunity, vol. 10, no. 5, pp. 373-379, 2009.

[6] S. Alfadhli, "Influence of endothelial nitric oxide synthase gene intron-4 $27 \mathrm{bp}$ repeat polymorphism on its expression in autoimmune diseases," Disease Markers, vol. 34, no. 5, pp. 349356, 2013.

[7] Q. Lu, A. Wu, and B. C. Richardson, "Demethylation of the same promoter sequence increases CD70 expression in lupus T cells and T cells treated with lupus-inducing drugs," The Journal of Immunology, vol. 174, no. 10, pp. 6212-6219, 2005.

[8] Q. Lu, A. Wu, L. Tesmer, D. Ray, N. Yousif, and B. Richardson, "Demethylation of CD40LG on the inactive $\mathrm{X}$ in $\mathrm{T}$ cells from women with lupus," The Journal of Immunology, vol. 179, no. 9, pp. 6352-6358, 2007.

[9] N. Hu, X. Qiu, Y. Luo et al., "Abnormal histone modification patterns in lupus CD4+ T cells," The Journal of Rheumatology, vol. 35, no. 5, pp. 804-810, 2008.

[10] T. Rauen, C. M. Hedrich, Y.-T. Juang, K. Tenbrock, and G. C. Tsokos, "cAMP-responsive element modulator (CREM) $\alpha$ protein induces interleukin 17A expression and mediates epigenetic alterations at the interleukin-17A gene locus in patients with systemic lupus erythematosus," The Journal of Biological Chemistry, vol. 286, no. 50, pp. 43437-43446, 2011.

[11] Y. Dai, Y.-S. Huang, M. Tang et al., "Microarray analysis of microRNA expression in peripheral blood cells of systemic lupus erythematosus patients," Lupus, vol. 16, no. 12, pp. 939946, 2007.

[12] Y. Tang, X. Luo, H. Cui et al., "MicroRNA-146a contributes to abnormal activation of the type I interferon pathway in human lupus by targeting the key signaling proteins," Arthritis \& Rheumatism, vol. 60, no. 4, pp. 1065-1075, 2009.

[13] C. M. Hedrich, T. Rauen, S. A. Apostolidis et al., "Stat3 promotes IL-10 expression in lupus T cells through trans-activation and chromatin remodeling," Proceedings of the National Academy of Sciences of the United States of America, vol. 111, no. 37, pp. 1345713462, 2014.

[14] A. Jönsen, A. Bengtsson, O. Nived, L. Truedsson, and G. Sturfelt, "Gene-environment interactions in the aetiology of systemic lupus erythematosus," Autoimmunity, vol. 40, no. 8, pp. 613-617, 2007.

[15] J. Cameron and J. Stewart, "Lupus nephritis," Journal of the American Society of Nephrology, vol. 10, no. 2, pp. 413-424, 1999.

[16] A. Gigante, M. L. Gasperini, A. Afeltra et al., "Cytokines expression in SLE nephritis," European Review for Medical and Pharmacological Sciences, vol. 15, no. 1, pp. 15-24, 2011.

[17] L. M. Ortega, D. R. Schultz, O. Lenz, V. Pardo, and G. N. Contreras, "Lupus nephritis: pathologic features, epidemiology and a guide to therapeutic decisions," Lupus, vol. 19, no. 5, pp. 557-574, 2010.

[18] G. S. Hill, M. Delahousse, D. Nochy, C. Mandet, and J. Bariéty, "Proteinuria and tubulointerstitial lesions in lupus nephritis," Kidney International, vol. 60, no. 5, pp. 1893-1903, 2001.

[19] A. Zickert, B. Sundelin, E. Svenungsson, and I. Gunnarsson, "Role of early repeated renal biopsies in lupus nephritis," Lupus Science \& Medicine, vol. 1, Article ID e000018, 2014. 
[20] D. D. Gladman, D. Ibañez, and M. B. Urowltz, "Systemic lupus erythematosus disease activity index 2000," The Journal of Rheumatology, vol. 29, no. 2, pp. 288-291, 2002.

[21] C. Lässer, V. Seyed Alikhani, K. Ekström et al., "Human saliva, plasma and breast milk exosomes contain RNA: uptake by macrophages," Journal of Translational Medicine, vol. 9, article 9, 2011.

[22] E. Lázaro-Ibáñez, A. Sanz-Garcia, T. Visakorpi et al., "Different gDNA content in the subpopulations of prostate cancer extracellular vesicles: apoptotic bodies, microvesicles, and exosomes," The Prostate, vol. 74, no. 14, pp. 1379-1390, 2014.

[23] M. Yáñez-Mó, P. R.-M. Siljander, Z. Andreu et al., "Biological properties of extracellular vesicles and their physiological functions," Journal of Extracellular Vesicles, vol. 4, Article ID 27066, 2015.

[24] S. A. Bellingham, B. M. Coleman, and A. F. Hill, "Small RNA deep sequencing reveals a distinct miRNA signature released in exosomes from prion-infected neuronal cells," Nucleic Acids Research, vol. 40, no. 21, pp. 10937-10949, 2012.

[25] H. Valadi, K. Ekström, A. Bossios, M. Sjöstrand, J. J. Lee, and J. O. Lötvall, "Exosome-mediated transfer of mRNAs and microRNAs is a novel mechanism of genetic exchange between cells," Nature Cell Biology, vol. 9, no. 6, pp. 654-659, 2007.

[26] E. Cocucci, G. Racchetti, and J. Meldolesi, "Shedding microvesicles: artefacts no more," Trends in Cell Biology, vol. 19, no. 2, pp. 43-51, 2009.

[27] S. Mathivanan, J. W. E. Lim, B. J. Tauro, H. Ji, R. L. Moritz, and R. J. Simpson, "Proteomics analysis of A33 immunoaffinitypurified exosomes released from the human colon tumor cell line LIM1215 reveals a tissue-specific protein signature," Molecular \& Cellular Proteomics, vol. 9, no. 2, pp. 197-208, 2010.

[28] J. Kowal, M. Tkach, and C. Théry, "Biogenesis and secretion of exosomes," Current Opinion in Cell Biology, vol. 29, no. 1, pp. 116-125, 2014.

[29] J. C. Akers, D. Gonda, R. Kim, B. S. Carter, and C. C. Chen, "Biogenesis of extracellular vesicles (EV): exosomes, microvesicles, retrovirus-like vesicles, and apoptotic bodies," Journal of Neuro-Oncology, vol. 113, no. 1, pp. 1-11, 2013.

[30] J. Lötvall, A. F. Hill, F. Hochberg et al., "Minimal experimental requirements for definition of extracellular vesicles and their functions: a position statement from the International Society for Extracellular Vesicles," Journal of Extracellular Vesicles, vol. 3, Article ID 26913, 2014.

[31] C. Théry, S. Amigorena, G. Raposo, and A. Clayton, "UNIT 3.22 Isolation and characterization of exosomes from cell culture supernatants and biological fluids," in Current Protocols in Cell Biology, pp. 22-29, John Wiley \& Sons, 2006.

[32] A. Cheruvanky, H. Zhou, T. Pisitkun et al., "Rapid isolation of urinary exosomal biomarkers using a nanomembrane ultrafiltration concentrator," The American Journal of PhysiologyRenal Physiology, vol. 292, no. 5, pp. F1657-F1661, 2007.

[33] D. Wang and W. Sun, "Urinary extracellular microvesicles: isolation methods and prospects for urinary proteome," Proteomics, vol. 14, no. 16, pp. 1922-1932, 2014.

[34] J. Schageman, E. Zeringer, M. Li et al., “The complete exosome workflow solution: from isolation to characterization of RNA cargo," BioMed Research International, vol. 2013, Article ID 253957, 15 pages, 2013.

[35] L. Muller, C.-S. Hong, D. B. Stolz, S. C. Watkins, and T. L. Whiteside, "Isolation of biologically-active exosomes from human plasma," Journal of Immunological Methods, vol. 411, pp. 55-65, 2014.
[36] H. Kalra, R. J. Simpson, H. Ji et al., "Vesiclepedia: a compendium for extracellular vesicles with continuous community annotation," PLoS Biology, vol. 10, no. 12, Article ID e1001450, 2012.

[37] R. J. Simpson, H. Kalra, and S. Mathivanan, "ExoCarta as a resource for exosomal research," Journal of Extracellular Vesicles, vol. 1, 2012.

[38] D.-K. Kim, J. Lee, S. R. Kim et al., "EVpedia: a community web portal for extracellular vesicles research," Bioinformatics, vol. 31, no. 6, pp. 933-939, 2015.

[39] H. Huang, J. Evankovich, W. Yan et al., "Endogenous histones function as alarmins in sterile inflammatory liver injury through Toll-like receptor 9 in mice," Hepatology, vol. 54, no. 3, pp. 999-1008, 2011.

[40] F. G. Goh and K. S. Midwood, "Intrinsic danger: activation of Toll-like receptors in rheumatoid arthritis," Rheumatology, vol. 51, no. 1, Article ID ker257, pp. 7-23, 2012.

[41] M. Schiller, P. Heyder, S. Ziegler et al., "During apoptosis HMGB1 is translocated into apoptotic cell-derived membraneous vesicles," Autoimmunity, vol. 46, no. 5, pp. 342-346, 2013.

[42] E. Boilard, P. A. Nigrovic, K. Larabee et al., "Platelets amplify inflammation in arthritis via collagen-dependent microparticle production," Science, vol. 327, no. 5965, pp. 580-583, 2010.

[43] L. A. Truman, C. A. Ford, M. Pasikowska et al., "CX3CL $1 /$ fractalkine is released from apoptotic lymphocytes to stimulate macrophage chemotaxis," Blood, vol. 112, no. 13, pp. 50265036, 2008.

[44] N. Cloutier, S. Tan, L. H. Boudreau et al., "The exposure of autoantigens by microparticles underlies the formation of potent inflammatory components: the microparticle-associated immune complexes," EMBO Molecular Medicine, vol. 5, no. 2, pp. 235-249, 2013.

[45] A. J. Ullal and D. S. Pisetsky, "The role of microparticles in the generation of immune complexes in murine lupus," Clinical Immunology, vol. 146, no. 1, pp. 1-9, 2013.

[46] N. Mor-Vaknin, F. Kappes, A. E. Dick et al., "DEK in the synovium of patients with juvenile idiopathic arthritis: characterization of DEK antibodies and posttranslational modification of the DEK autoantigen," Arthritis \& Rheumatism, vol. 63, no. 2, pp. 556-567, 2011.

[47] L. E. Muñoz, C. Janko, G. E. Grossmayer et al., "Remnants of secondarily necrotic cells fuel inflammation in systemic lupus erythematosus," Arthritis and Rheumatism, vol. 60, no. 6, pp. 1733-1742, 2009.

[48] J. Dieker, L. Hilbrands, A. Thielen, H. Dijkman, J. H. Berden, and J. van der Vlag, "Enhanced activation of dendritic cells by autologous apoptotic microvesicles in MRL/lpr mice," Arthritis Research \& Therapy, vol. 17, article 103, 2015.

[49] C. T. Nielsen, O. Østergaard, C. Johnsen, S. Jacobsen, and N. H. H. Heegaard, "Distinct features of circulating microparticles and their relationship to clinical manifestations in systemic lupus erythematosus," Arthritis \& Rheumatism, vol. 63, no. 10, pp. 3067-3077, 2011.

[50] O. Østergaard, C. T. Nielsen, L. V. Iversen et al., "Unique protein signature of circulating microparticles in systemic lupus erythematosus," Arthritis \& Rheumatism, vol. 65, no. 10, pp. 2680-2690, 2013.

[51] J. R. Dye, A. J. Ullal, and D. S. Pisetsky, “The role of microparticles in the pathogenesis of rheumatoid arthritis and systemic lupus erythematosus," Scandinavian Journal of Immunology, vol. 78, no. 2, pp. 140-148, 2013. 
[52] E. K. Kapsogeorgou, R. F. Abu-Helu, H. M. Moutsopoulos, and M. N. Manoussakis, "Salivary gland epithelial cell exosomes: a source of autoantigenic ribonucleoproteins," Arthritis \& Rheumatism, vol. 52, no. 5, pp. 1517-1521, 2005.

[53] B. Parker, A. Al-Husain, P. Pemberton et al., "Suppression of inflammation reduces endothelial microparticles in active systemic lupus erythematosus," Annals of the Rheumatic Diseases, vol. 73, no. 6, pp. 1144-1150, 2014.

[54] T. Pisitkun, R.-F. Shen, and M. A. Knepper, "Identification and proteomic profiling of exosomes in human urine," Proceedings of the National Academy of Sciences of the United States of America, vol. 101, no. 36, pp. 13368-13373, 2004.

[55] K. C. Miranda, D. T. Bond, M. McKee et al., "Nucleic acids within urinary exosomes/microvesicles are potential biomarkers for renal disease," Kidney International, vol. 78, no. 2, pp. 191$199,2010$.

[56] F. Barutta, M. Tricarico, A. Corbelli et al., "Urinary exosomal microRNAs in incipient diabetic nephropathy," PLoS ONE, vol. 8, no. 11, Article ID e73798, 2013.

[57] H. Zhou, A. Cheruvanky, X. Hu et al., "Urinary exosomal transcription factors, a new class of biomarkers for renal disease," Kidney International, vol. 74, no. 5, pp. 613-621, 2008.

[58] S. Bala, J. Petrasek, S. Mundkur et al., "Circulating microRNAs in exosomes indicate hepatocyte injury and inflammation in alcoholic, drug-induced, and inflammatory liver diseases," Hepatology, vol. 56, no. 5, pp. 1946-1957, 2012.

[59] L. Cheng, X. Sun, B. J. Scicluna, B. M. Coleman, and A. F. Hill, "Characterization and deep sequencing analysis of exosomal and non-exosomal miRNA in human urine," Kidney International, vol. 86, no. 2, pp. 433-444, 2014.

[60] O. Ichii, S. Otsuka-Kanazawa, T. Horino et al., "Decreased miR-26a expression correlates with the progression of podocyte injury in autoimmune glomerulonephritis," PLoS ONE, vol. 9, no. 10, Article ID e110383, 2014.

[61] C. Solé, J. Cortés-Hernández, M. L. Felip, M. Vidal, and J. OrdiRos, "miR-29c in urinary exosomes as predictor of early renal fibrosis in lupus nephritis," Nephrology Dialysis Transplantation, vol. 30, no. 9, pp. 1488-1496, 2015.

[62] Y. Tang, X. Luo, H. Cui et al., "MicroRNA-146a contributes to abnormal activation of the type I interferon pathway in human lupus by targeting the key signaling proteins," Arthritis and Rheumatism, vol. 60, no. 4, pp. 1065-1075, 2009.

[63] G. Wang, L.-S. Tam, E. K.-M. Li et al., "Serum and urinary cell-free MiR-146a and MiR-155 in patients with systemic lupus erythematosus," The Journal of Rheumatology, vol. 37, no. 12, pp. 2516-2522, 2010.

[64] H. Wang, W. Peng, X. Ouyang, W. Li, and Y. Dai, "Circulating microRNAs as candidate biomarkers in patients with systemic lupus erythematosus," Translational Research, vol.160, no. 3, pp. 198-206, 2012.

[65] J. Lu, B. C.-H. Kwan, F. M.-M. Lai et al., "Glomerular and tubulointerstitial miR-638, miR-198 and miR-146a expression in lupus nephritis," Nephrology, vol. 17, no. 4, pp. 346-351, 2012.

[66] S. E. Löfgren, J. Frostegård, L. Truedsson et al., "Genetic association of miRNA-146a with systemic lupus erythematosus in Europeans through decreased expression of the gene," Genes and Immunity, vol. 13, no. 3, pp. 268-274, 2012.

[67] L.-L. Lv, Y.-H. Cao, M.-M. Pan et al., "CD2AP mRNA in urinary exosome as biomarker of kidney disease," Clinica Chimica Acta, vol. 428, pp. 26-31, 2014.
[68] S. Spanu, C. R. C. van Roeyen, B. Denecke, J. Floege, and A. S. Mühlfeld, "Urinary exosomes: a novel means to non-invasively assess changes in renal gene and protein expression," PLOS ONE, vol. 9, no. 10, Article ID e109650, 2014.

[69] P. Gutwein, A. Schramme, M. S. Abdel-Bakky et al., "ADAM10 is expressed in human podocytes and found in urinary vesicles of patients with glomerular kidney diseases," Journal of Biomedical Science, vol. 17, no. 1, article 3, 2010.

[70] T. Niranjan, B. Bielesz, A. Gruenwald et al., “The Notch pathway in podocytes plays a role in the development of glomerular disease," Nature Medicine, vol. 14, no. 3, pp. 290-298, 2008.

[71] K. Al-Nedawi, B. Meehan, J. Micallef et al., "Intercellular transfer of the oncogenic receptor EGFRvIII by microvesicles derived from tumour cells," Nature Cell Biology, vol. 10, no. 5, pp. 619-624, 2008.

[72] M. F. Baietti, Z. Zhang, E. Mortier et al., "Syndecan-synteninALIX regulates the biogenesis of exosomes," Nature Cell Biology, vol. 14, no. 7, pp. 677-685, 2012.

[73] A. Bobrie, S. Krumeich, F. Reyal et al., "Rab27a supports exosome-dependent and -independent mechanisms that modify the tumor microenvironment and can promote tumor progression," Cancer Research, vol. 72, no. 19, pp. 4920-4930, 2012.

[74] D. Sun, X. Zhuang, X. Xiang et al., "A novel nanoparticle drug delivery system: the anti-inflammatory activity of curcumin is enhanced when encapsulated in exosomes," Molecular Therapy, vol. 18, no. 9, pp. 1606-1614, 2010.

[75] G.-J. Wang, Y. Liu, A. Qin et al., "Thymus exosomes-like particles induce regulatory T cells," The Journal of Immunology, vol. 181, no. 8, pp. 5242-5248, 2008.

[76] S.-H. Kim, E. R. Lechman, N. Bianco et al., "Exosomes derived from IL-10-treated dendritic cells can suppress inflammation and collagen-induced arthritis," The Journal of Immunology, vol. 174, no. 10, pp. 6440-6448, 2005.

[77] S. H. Kim, N. Bianco, R. Menon et al., "Exosomes derived from genetically modified DC expressing FasL are anti-inflammatory and immunosuppressive," Molecular Therapy, vol. 13, no. 2, pp. 289-300, 2006.

[78] L. Alvarez-Erviti, Y. Seow, H. Yin, C. Betts, S. Lakhal, and M. J. A. Wood, "Delivery of siRNA to the mouse brain by systemic injection of targeted exosomes," Nature Biotechnology, vol. 29, no. 4, pp. 341-345, 2011.

[79] N. Cloutier, A. Paré, R. W. Farndale et al., "Platelets can enhance vascular permeability," Blood, vol. 120, no. 6, pp. 1334-1343, 2012. 


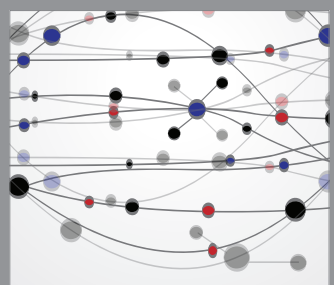

The Scientific World Journal
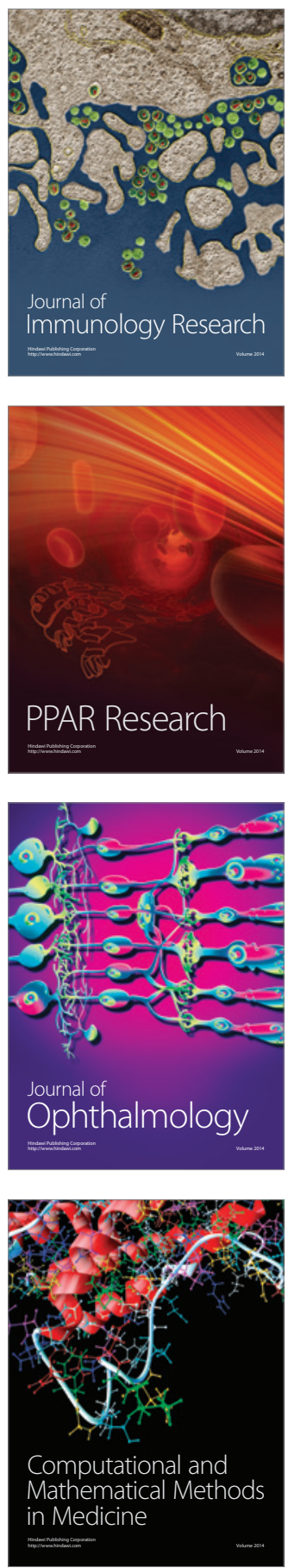

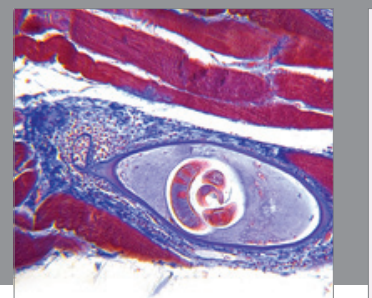

Gastroenterology

Research and Practice
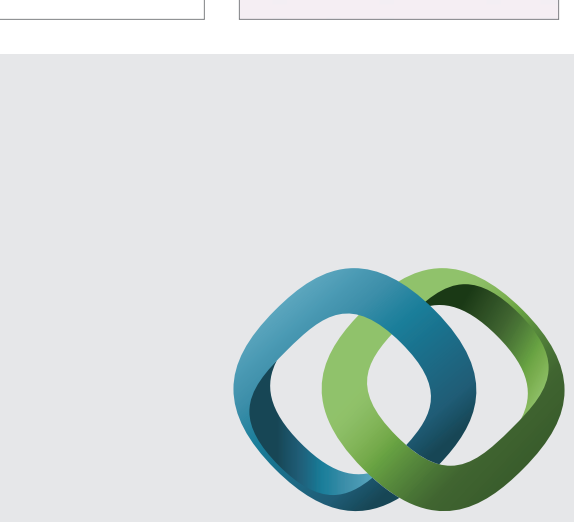

\section{Hindawi}

Submit your manuscripts at

http://www.hindawi.com
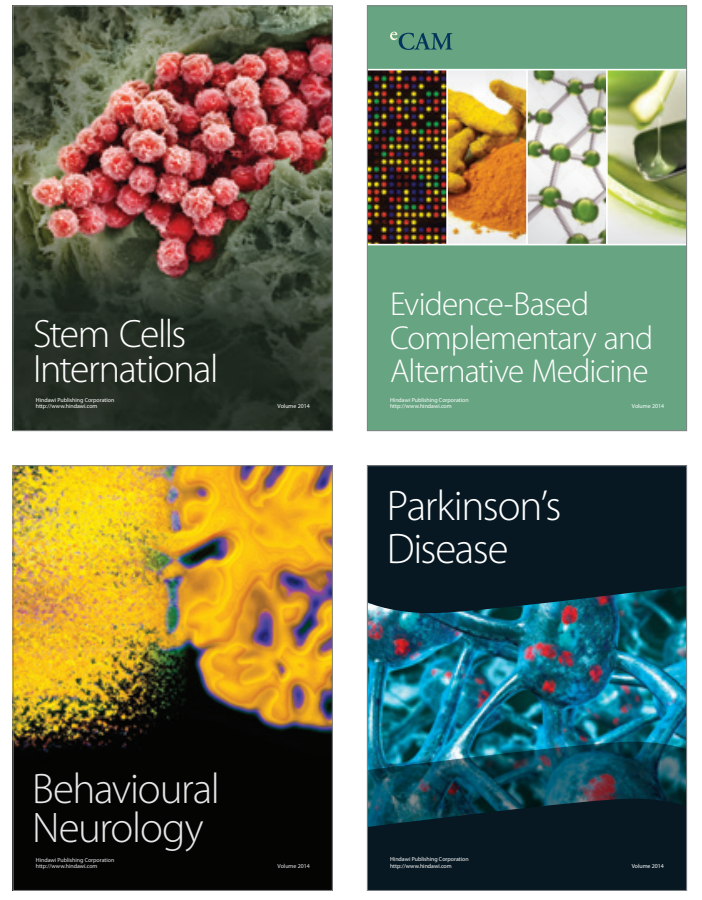
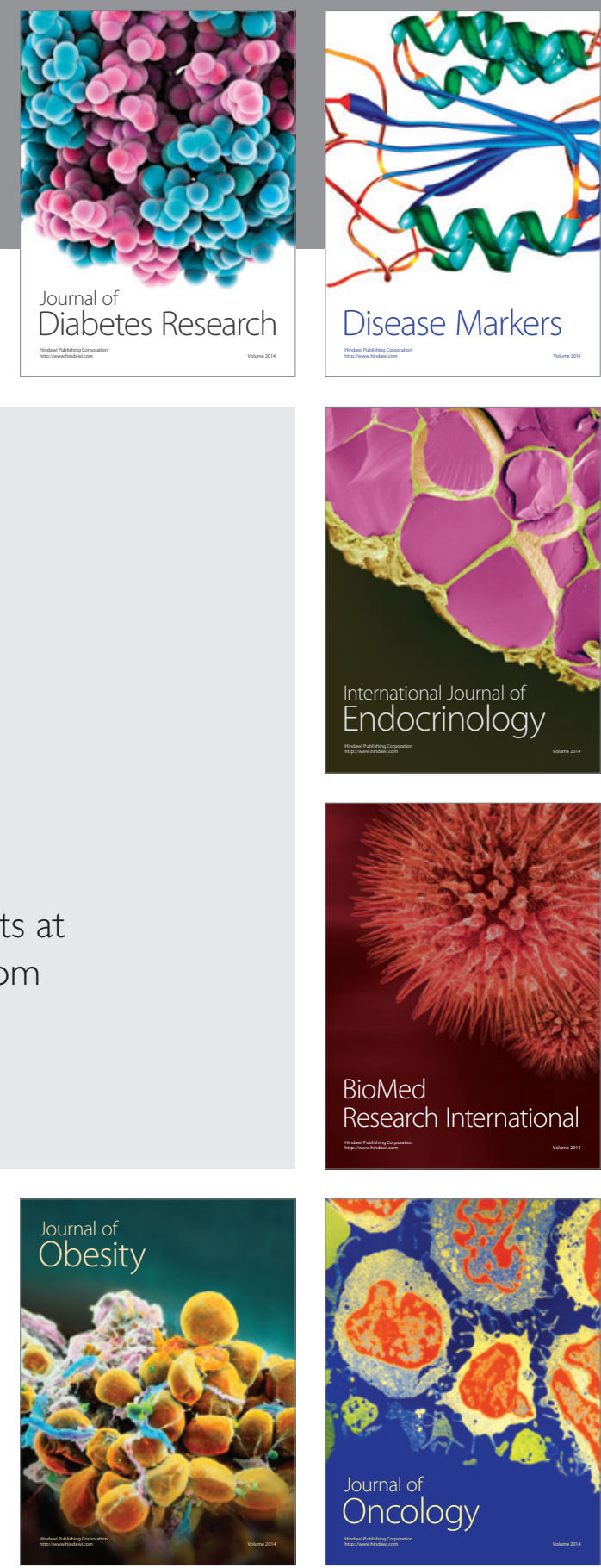

Disease Markers
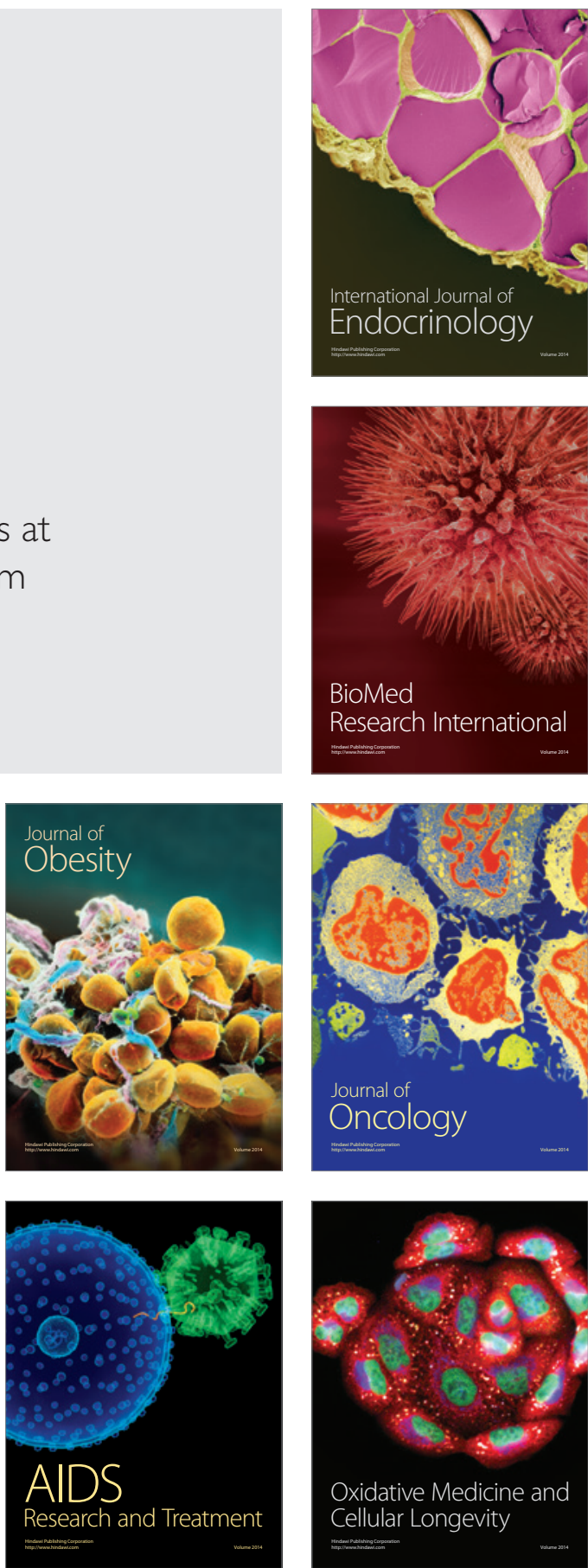